\title{
Corrosion Diagnosis of a Ship Mock-Up From Near Electric-Field Measurements
}

\author{
Arnaud Guibert ${ }^{1}$, Jean-Louis Coulomb ${ }^{1}$, Olivier Chadebec ${ }^{1}$, and Corinne Rannou ${ }^{2}$ \\ ${ }^{1}$ Grenoble Electrical Engineering Lab Grenoble-INP/Université Joseph Fourier/CNRS, Grenoble 38402, France \\ ${ }^{2}$ GESMA-DGA, Brest 29240, France
}

\begin{abstract}
We present here an original application linking an electrochemical phenomenon and the computational aspect of electromagnetic fields to provide a corrosion diagnosis of a protected underwater steel structure. This is done with the pairing of a boundary element method and the resolution of an inverse problem. After a defined operating time, it is mandatory to check an underwater steel structure. Sadly, current examinations techniques require immobilizing the structure for a long time and are less efficient. The purpose of this paper is to replace this checking by a series of close electrical measurements in the conducting water which provides a corrosion diagnosis of the structure. The new method introduced ensures great time-savings but also an accuracy never reached before. This paper presents this numerical method and its checking through real electrical measurements on a frigate mock-up.
\end{abstract}

Index Terms-Boundary element method (BEM), cathodic protection, inverse problems, regularization techniques.

\section{INTRODUCTION}

D URING its operating life, an underwater structure suffers from corrosion. This phenomenon starts when iron paint defects appear on its surface, electrically linked to noble metals (propellers in bronze and nickel for example). This reaction, called galvanic coupling, makes the iron an anode and bronze a cathode. To fight against this reaction, two main methods have been developed [1] as follows.

1) The sacrificial anode cathodic protection (SACP): some less noble elements than iron (zinc, aluminium, etc....) are placed on the structure to protect it. They are going to be corroded instead of the iron of the structure itself, becoming the new anode of the reaction.

2) The impressed current cathodic protection (ICCP): Platinum anodes are distributed on the structure, injecting currents in the seawater. This places the iron in its passivation zone and protects it.

These two protections lead to a circulation of currents in the seawater, inducing the presence of a varying electromagnetic field. The first step of the study is to predict this electromagnetic field from the electric boundary conditions (taken from the electrochemical context). Then, the problem will be inversed and corrosion diagnosis will be deducted, starting from near electric-field measurements and leading to the corroded areas localization. This paper, based on electric fields and applied to real measurements on a mock-up, extensively improves a method based on electric potential measurements, previously introduced [2].

\section{FORWARD MODELING}

As stated before, the forward modeling aims to predict the electric potential and the electromagnetic field from physic boundary conditions. Since the main goal is to achieve a diagnosis tool, the numeric method used must directly link

Manuscript received December 21, 2009; revised March 09, 2010; accepted April 06, 2010. Current version published July 21, 2010. Corresponding author:

A. Guibert (e-mail: arnaud.guibert@g2elab.inpg.fr).

Color versions of one or more of the figures in this paper are available online at http://ieeexplore.ieee.org.

Digital Object Identifier 10.1109/TMAG.2010.2048098 the boundary conditions to the measurement locations, an advantage provided by the boundary element method (BEM). Moreover, this method permits a simple modeling of the infinity region [3], [4].

Starting from the Laplace equation, the principle is to use the third Green's identity to write an integral equation on the $\Gamma$ boundaries of the problem

$$
\begin{aligned}
h(M) \cdot \varphi(M)+\oint_{\partial \Gamma} \varphi(P) & \cdot \frac{\partial}{\partial n_{q}} G(M, P) \cdot d l \\
& \quad-\oint_{\partial \Gamma} G(M, P) \cdot \frac{\partial}{\partial n_{q}} \varphi(P) d l=0
\end{aligned}
$$

where $\varphi$ is the electric potential and $\partial \varphi / \partial \mathrm{n}$ is the opposite of the normal current density, within the conductivity $\sigma$ of the domain. $\mathrm{P}$ is the integration point mobile on the boundary and $\mathrm{M}$ is the point where the computation is made. $\mathrm{h}(\mathrm{M})$ is the solid angle seen by $\mathrm{M}$ and $\mathrm{G}$ is the Green function in 3-D (1/r, where $\mathrm{r}$ is the distance $|\mathrm{MP}|)$.

After the surfaces have been meshed in $N$ elements, all unknowns are considered constant on each element by a zero-order approximation. Then, a point matching approach of (1) is provided, setting the unknowns at the barycenter of each element. This numerical stage leads to write (1) as the following matrix system:

$$
\left[A_{\text {Green }}\right] \cdot\left[\begin{array}{c}
\varphi \\
\frac{\partial \varphi}{\partial n}
\end{array}\right]=0
$$

Making this approximation is known to not be very precise, but since the main goal is the development of an inverse method, it is acceptable for us. Of course, this system has more unknowns than equations. Solving it requires the introduction of the boundary conditions (a part of the $(\varphi, \partial \varphi / \partial \mathrm{n})$ ).

For the last step of this forward modeling, we obtain a nonlinear system due to the form of the polarization law $(\partial \varphi / \varphi \mathrm{n}=$ $f(\varphi)$ ) introduced by the presence of polarizable steel on the boundaries. This system is then usually solved by an iterative Newton-Raphson process. Some results are presented in [2] on a simple ship mock-up: with parts of the boundary conditions on the structure (potential of SACP anodes, current densities of ICCP anodes, isolated parts, and polarization laws of cathodes), and this algorithm finds the missing ones. 
Once all of the boundary conditions are obtained, it is possible to compute the electric field anywhere in the domain thanks to the gradient of (1)

$$
\mathbf{E}(M)=\frac{1}{4 \pi} \cdot \oint_{\partial \Gamma} \varphi(P)\left(-3 \cdot \frac{(\mathbf{r} . \mathbf{n}) \cdot \mathbf{r}}{r^{5}}+\frac{\mathbf{n}}{r^{3}}\right)+\frac{\partial \varphi(P)}{\partial n} \cdot \frac{\mathbf{r}}{r^{3}} \cdot d l .
$$

The last analytical expression shows two integral terms to compute, which is again too difficult for complex geometries. The new numerical expression becomes in 3-D

$$
\begin{aligned}
\mathbf{E}(M) & =\frac{1}{4 \pi} \cdot \sum_{j=1}^{N} \varphi_{j}\left(Q_{j}\right) \cdot \iint_{S_{j}(\Omega)}\left(-3 \cdot \frac{\left(\mathbf{r}_{\mathbf{i j}} \cdot \mathbf{n}\right) \cdot \mathbf{r}_{\mathbf{i j}}}{r_{i j}^{5}}+\frac{\mathbf{n}}{r_{i j}^{3}}\right) \cdot d S_{j}(\Omega) \\
& -\frac{1}{4 \pi} \cdot \sum_{j=1}^{N} \frac{\partial \varphi_{j}\left(Q_{j}\right)}{\partial n_{j}} \cdot \iint_{S_{j}(\Omega)}-\frac{\mathbf{r}_{\mathbf{i j}}}{r_{i j}^{3}} \cdot d S_{j}(\Omega) .
\end{aligned}
$$

The $\varphi_{\mathrm{j}}$ and $\partial \varphi_{\mathrm{j}} / \partial \mathrm{n}$ on the boundaries have been obtained by the previous Newton algorithm. In this case, there are only $2 N$ analytical integrals to compute. This is accomplished by a numerical approximation with Gauss points (there is no singularity if the calculation point $M$ is not on the boundaries).

If the electric field is expressed in several points, the last equation can be represented by the following system, which is the basis of the inverse method:

$$
\left[A_{\text {Measurement }}\right] \cdot\left[\begin{array}{c}
\varphi \\
\frac{\partial \varphi}{\partial n}
\end{array}\right]=[E] .
$$

\section{INVERSE METHOD AND IMPROVEMENTS}

The main goal is, from real electromagnetic measurements, contained in the $\mathrm{E}$ vector and the construction of the system (5) to find the source vector $(\varphi$ and $\partial \varphi / \partial \mathrm{n})$. When trying to directly inverse this system with a least squares method, the solution obtained is very bad, with quantities reaching huge values $\left(10^{14}\right.$ while expecting potentials close to the decade of Volts for example). This problem is very ill posed (very bad condition number) due to two main reasons:

1) A mathematical one: most systems have fewer lines than rows (more unknowns than equations) and it has infinity of solutions. Another reason damaging the condition number is the error introduced by the computational approximation of the matrix $\mathrm{A}_{\text {Measurement }}$ coefficients (done during build of the numerical system (5)). Finally, the measurement errors in the $\mathbf{E}$ vector deteriorate it.

2) A physical one: the build of the system (5) is made with the expressions of electric-field creation by charges and dipoles. When directly inversing (5), the solution obtained is charges and dipoles decorrelated, which do not guarantee the third Green's identity.

A good way to solve the physical difficulty is to add Green's equations from (2), linking all unknowns on the boundaries. This ensures the solution to fit to the 3rd Green's identity. Dipoles now fit potentials and charges fit their normal derivative. We have considerably reduced the solution space of

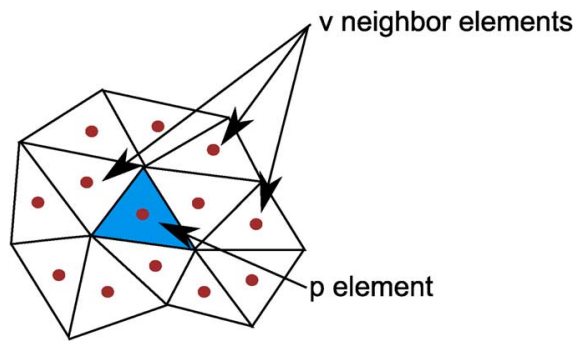

Fig. 1. Representation of simple meshing.

research. The new system that has been obtained can be written by the following expression:

$$
\left[\begin{array}{c}
A_{\text {Measurement }} \\
A_{\text {Green }}
\end{array}\right] \cdot\left[\begin{array}{c}
\varphi \\
\frac{\partial \varphi}{\partial n}
\end{array}\right]=\left[\begin{array}{c}
E \\
0
\end{array}\right] .
$$

Since we have a series of zeros on the right member, this system can be simplified by eliminating one kind of unknown. This choice is then deducted from the information we have on the anodes: for an ICCP case, their current density is known, so we have to suppress the potentials from the system defined in (5). For SACP cases, the anodes potential is known and we have to simplify the current densities. The new system can be finally written

$$
[A] \cdot[X]=[B] .
$$

In both cases, the final system has as many equations as measurement points and as many unknowns as meshing elements. Finally, as we logically want to have more equations than unknowns (more lines than rows) to ensure the solution unicity, we can choose the number of measurements to obtain from the meshing knowledge of the structure. This does not significantly improve the condition number but gives a physical behavior to the solution: indeed, quantities obtained guarantee the third Green's identity and they empirically have amplitudes close to the expected ones but with a bad repartition.

Then, we have to deal with the mathematical difficulty. The new system now has more lines than rows and only one kind of source to find, so there is no solution. We have to choose the best approximated solution. This can be performed thanks to regularization techniques, such as truncation of the singular value decomposition (TSVD). This technique avoids taking the more noisy values into account. Tikhonov's method is preferred since it allows imposing a chosen physical behavior to the solution. Its principle is to find a solution minimizing the following equation [5]:

$$
\min (\|A . X-B\|+\lambda .\|L \cdot X\|) .
$$

The $L$ matrix is called the regularization matrix and imposes the physical behavior: zero-order regularization ( $L$ is the identity matrix and has an $N$ dimension) privileges solutions with minimum norm; a first order one favors continuous solutions (minimizing the gradient) and a second-order one accentuates this continuous behavior (minimizing the Laplacian). In our study, a first-order method is chosen. To take a visible example, let us consider a meshing part: 


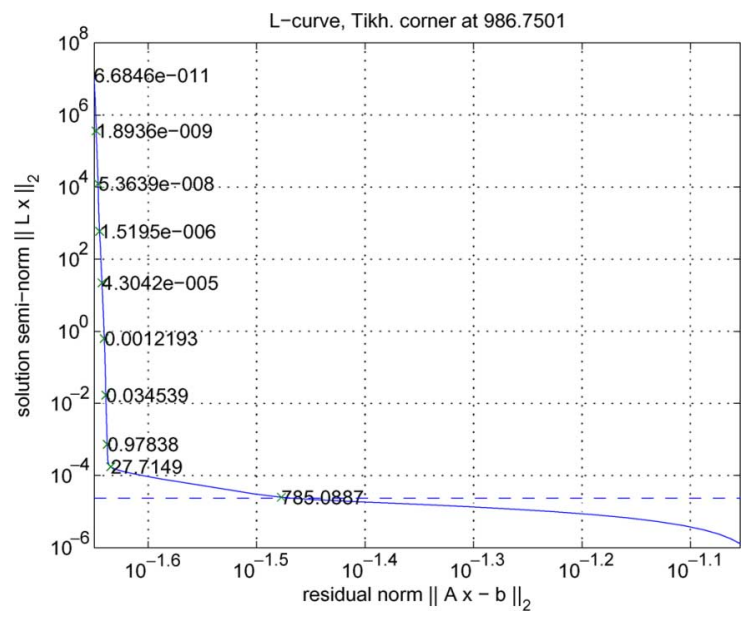

Fig. 2. Classical kind of L-curve.

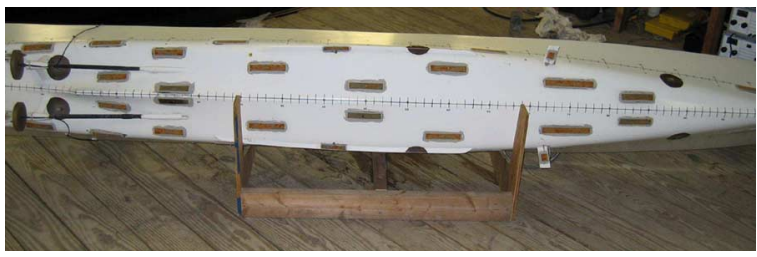

Fig. 3. The 1/40th mock-up with 36 defects and ICCP.

The goal is to minimize the value difference between the $p$ element and its neighbors. This is done by searching the $v$ neighbors of $p$ and affecting them with the $1 / v$ coefficient on the $p$ line of the $L$ matrix

$$
\mathbf{L}_{\mathbf{p}}=\left[\begin{array}{llllllllllllll}
0 & \ldots & 0 & \frac{1}{v} & \ldots & \frac{1}{v} & -1 & \frac{1}{v} & \ldots & \frac{1}{v} & 0 & \ldots & 0
\end{array}\right]
$$

The $\lambda$ choice is then the heart of the inverse method. It symbolizes the importance given to the regularization compared to the mathematical precision. One well-known tool to observe this compromise is the L-Curve: for several $\lambda$ values [5], the residual Euclidian norm $\|\mathrm{A} . \mathrm{X}-\mathrm{B}\|_{2}$ is traced on the abscissas axe and the solution semi-norm $\|\mathrm{L} . \mathrm{X}\|_{2}$ on the ordinate one. This curve has often an $L$ shape and the best $\lambda$ area corresponds to its corner, which minimizes residual and solution norms. Let us take a classical example of the L-Curve obtained with simple geometry and measurements without noise

The corner can be easily found graphically and then we have to choose the best parameter, privileging the residual or the solution norm. An important remark to take into account is that we find the corrosion areas with a graphical analysis of the solution. That is why we prefer having a solution with more approximate amplitudes but a better repartition of them than the contrary. Finally, in most cases, the priority is given to the minimization of the solution seminorm.

\section{Method Checking With Real Measurements}

To check the diagnosis method explained before, real measurements are employed: a composite 1/40th mock-up of a frigate has been equipped with an adapted ICCP (with six platinum anodes), electrically linked to iron plates (simulating the paint defects) disposed on the hull.

The mock-up is then placed in salt water with a controlled conductivity $(5,16 \mathrm{~S} / \mathrm{m})$ and bubbling to simulate real navigating conditions.

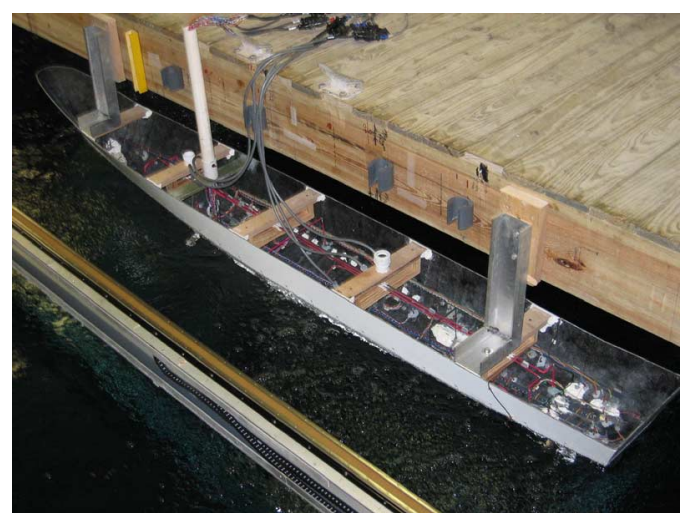

Fig. 4. The 1/40th mock-up set in measurement conditions.

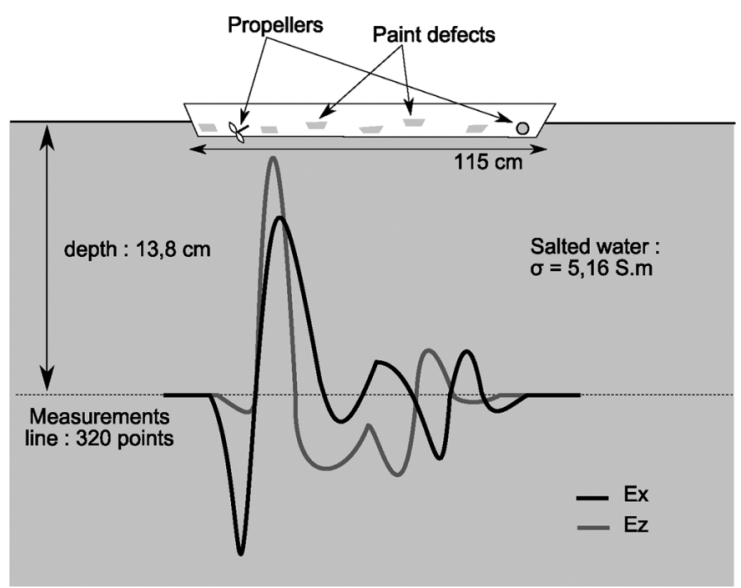

Fig. 5. Real electromagnetic measurements on a 1/40th mock-up.

When the ICCP is running, some longitudinal electric-field measurements are made on lines at two different depths:

These measurements have been made in physical scale modeling (PSM) in the facility of the U.S. Naval Research Laboratory (NRL) in Key West, FL. In fact, 640 measurements are made: $320 \mathrm{E}_{\mathrm{x}}$ and $320 \mathrm{E}_{\mathrm{z}}$, and not one is made on the $y$ (transverse) axis. Indeed, as the geometry is quasisymmetric in the longitudinal axis, there is very less transverse field under the keel. The complex meshing of the structure gives 3103 elements, making the system underdetermined. Fewer elements is due to the numerical limitation of BEM use.

Since we are in an ICCP case, the potential has to be simplified to only keep the current densities as unknown. The 3103 Green equations are injected and the knowledge of the anodes current density (in the range of a hundred $\mathrm{A} / \mathrm{m}^{2}$ ) gives a new system with 640 equations and 3087 current density unknowns. We have too few equations to ensure the solution unicity so the decision is made to interpolate some more values from the measurements to obtain 3300 final values. This operation could be problematic because useful values not appearing in the first graph will be ignored with this method. Moreover, it can introduce redundancy, deteriorating the condition number of the system.

The final system now has 3300 equations and 3087 unknowns which make it overdetermined, and the unicity of the solution is ensured. The regularization technique is the Tikhonov one with a zero order. We do not use a first-order regularization in this example, because of the presence of the propellers. Indeed, this method gives very good results when the measurement points 

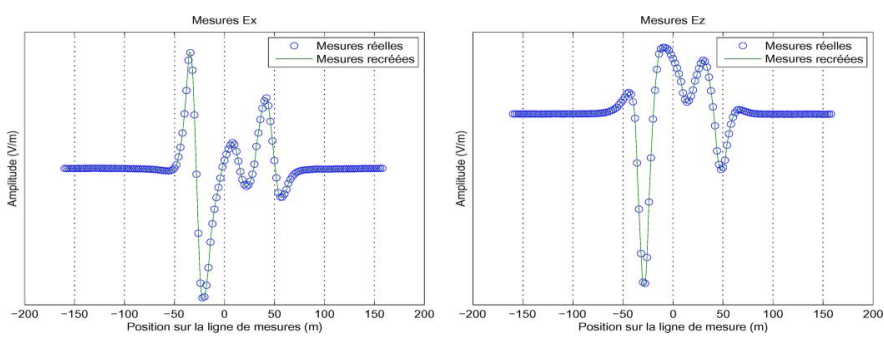

Fig. 6. Measurements interpolation.

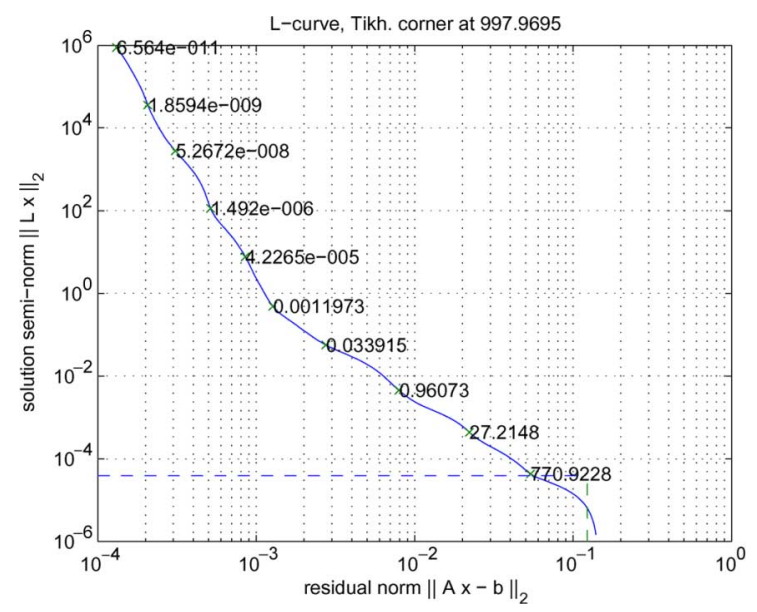

Fig. 7. L-curve obtained with the 0-order regularization.

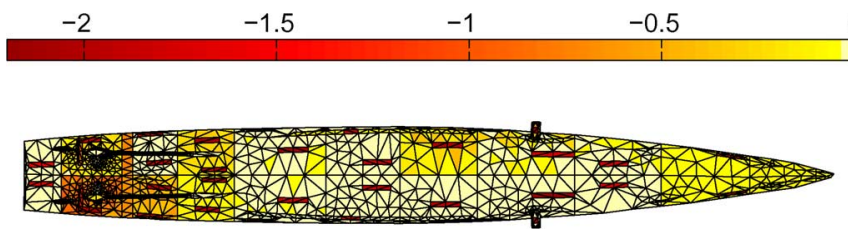

Fig. 8. Current density obtained on the boundaries in $\mathrm{A} / \mathrm{m}^{2}$.

are well situated. This is not the case here at the rear of the hull because some defects appear between the hull and the propellers. Reducing the gradient of current densities in this area from measurements points situated below propellers completely erases locating defects. This shows the importance of thinking about the measurements number and position.

Using zero-order regularization leads to the following L-Curve:

The curve does not have an $\mathrm{L}$ shape and the $\lambda$ choice is not obvious. As stated before, we prefer to choose a more regularized solution (to minimize the solution) toward the mathematical precision. Moreover, we can graphically constrain the area of the $\lambda$ search with the knowledge of the current density injected in the water. This imposes an upper bound to the solution seminorm (remember, we use zero-order regularization), that is to say, a maximum current density admissible on the hull.

The parameter is chosen to be equal to 27 , and the current density results obtained on the hull are in the following section (only negative ones are represented, which corresponds to the ones going back to the hull; positive ones are the injected):

Those results should be compared to the defects repartition in Fig. 1. The corroded areas correspond to the areas where the current density goes back to the hull with the maximum amplitude and most of them fit the expected repartition. The only area where the result is not explicit is the rear part of the hull, under

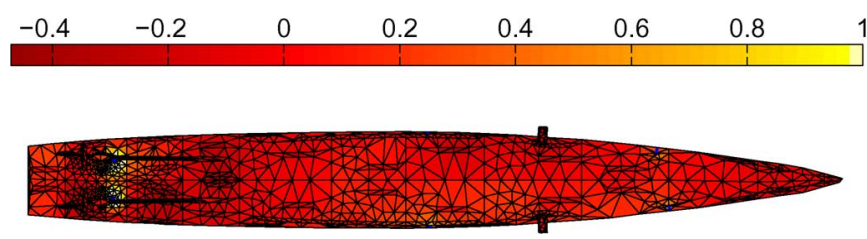

Fig. 9. Potential obtained on the boundaries in volts.

the propellers, which considerably perturb the diagnosis. Mathematically, the result matches the real location of the iron plates, giving a good diagnosis, with less than 5\% error. This global error is evaluated by computing the sum of the local root mean square (rms) difference between the expected value (obtained by forward modeling) and the diagnosis value. Once those current densities are obtained, it is possible to get the potentials on the hull with (2).

The other visible proof of a corroded area is the lowest potential zone, but it is not very relevant in most cases. Indeed, the definition of the lowest potential has to be taken from a percentage of the total difference between potential extremes, difficult to clearly express in general. The corrosion diagnosis based on a current density study is more relevant. That is why in SACP cases (where the diagnosis is made on potentials), the current density reconstruction with (2) is necessary.

\section{CONCLUSION}

This diagnosis method globally succeeds in locating the corroded areas of an immerged part of a hull with real electric-field measurements. Previous work was based on an electric potential study, giving also good theoretical and empirical results [2]. These two approaches of the work do not have the same advantages, because of their own decreasing behavior in a conducting region. Indeed, a field approach is less influenced by perturbations but is more difficult to measure far away from the structure (its decrease follows a $1 / \mathrm{r}^{3}$ law) while the contrary is true for the potentials (following a $1 / \mathrm{r}^{2}$ law). Finally, the diagnosis of a structure begins with the study of the meshing used, imposing the number of measurements to perform. The type and localization of those measurements then have to be optimized to obtain the best results (best inverse matrix condition number). Some work can be done to increase the precision and numerical aspects. We can assume that the presented method used here will be able to be applied to other types of structures, such as pipelines, offshore wind turbines, or oilrigs.

\section{REFERENCES}

[1] J. Creus, R. Sabot, and P. Refait, "Corrosion et protection des métaux en milieu marin," Techniques de l'ingénieur, Corrosion et vieillissement, vol. COR1, no. COR620, pp. COR620.1-COR620.14, 2004.

[2] A. Guibert, J.-L. Coulomb, O. Chadebec, and C. Rannou, "Ships hull corrosion diagnosis from close measurements of electric potential in the water," IEEE Trans. Magn., vol. 45, 2009, to be published.

[3] J. A. Brebbia, J. C. F. Telles, and L. C. Wrobel, Boundary Elements Techniques, Theory and Applications in Engineering. Berlin, Germany: Springer-Verlag, 1984.

[4] N. G. Zamani, J. M. Chuang, and J. F. Porter, "BEM simulation of cathodic protection systems employed in infinite electrolytes," Int. J. Numer. Meth. Eng., vol. 24, pp. 605-620, 1987.

[5] C. Hansen, Rank-Deficient and Discrete Ill-Posed Problems, Numerical Aspects of Linear Inversion. Philadelphia, PA: SIAM, 1998.

[6] V. A. Morozov, "On the solution of functional equations by the method of regularization," Soviet Math. Dokl., vol. 7, pp. 414-417, 1966. 